\title{
Development and characterization of the replicon system of Japanese encephalitis live vaccine virus SA14-14-2
}

Shi-Hua Li ${ }^{\dagger}$, Xiao-Feng Li ${ }^{\dagger}$, Hui Zhao, Yong-Qiang Deng, Xue-Dong Yu, Shun-Ya Zhu, Tao Jiang, Qing Ye, E-De Qin and Cheng-Feng Qin ${ }^{*}$

\begin{abstract}
Background: Viral self-replicating sub-genomic replicons represent a powerful tool for studying viral genome replication, antiviral screening and chimeric vaccine development. Many kinds of flavivirus replicons have been developed with broad applications.

Findings: The replicon system of JEV live vaccine strain SA14-14-2 was successfully developed in this study. Two kinds of replicons that express enhanced green fluorescent protein (EGFP) and Renilla luciferase (R.luc) were constructed under the control of SP6 promoter, respectively. Robust EGFP and R.luc signals could be detected in the replicon-transfected BHK-21 cells. Furthermore, the potential effects of selected amino acids in the C-terminal of envelope protein on replication were characterized using the replicon system.
\end{abstract}

Conclusions: Our results provide a useful platform not only for the study of JEV replication, but also for antiviral screening and chimeric vaccine development.

Keywords: Japanese encephalitis virus (JEV), SA14-14-2, Replicon

Japanese encephalitis is now recognized as the leading cause of viral encephalitis in Asian countries, including China, Japan, Korea, the Philippines, Thailand, and India $[1,2]$. Clinical Japanese encephalitis is a severe disease with a high case fatality rate. World Health Organization (WHO) estimates that approximately 50,000 cases of Japanese encephalitis occur each year, resulting in about 10,000 deaths and 15,000 cases of neurological or psychiatric sequelae $[3,4]$. Japanese encephalitis virus (JEV) is transmitted in an enzootic cycle between Culex species mosquitoes and vertebrates, primarily birds with pigs serving as amplifying hosts. In recent years, JEV has begun to spread to other geographic areas such as Pakistan and Australia $[5,6]$. The geographic expansion and high fatality rates have drawn increasing attention from the international public health community [7].

\footnotetext{
* Correspondence: qincf@bmi.ac.cn

${ }^{\dagger}$ Equal contributors

Department of Virology, State Key Laboratory of Pathogen and Biosecurity, Beijing Institute of Microbiology and Epidemiology, Beijing 100071, China
}

Vaccination has been recognized as the most reliable and economic measure for protection against Japanese encephalitis. Currently, three kinds of vaccines are available: inactivated vaccine produced in mouse-brain or cell culture and live attenuated vaccine produced on primary hamster kidney (PHK) cells [8]. The live vaccine (SA1414-2) was initially licensed in 1989 in mainland China, and now exported to most JEV-endemic countries, including India, Sri Lanka, Nepal, Thailand and South Korea under the recommendation of WHO [9]. Large scale immunizations in more than 300 million children have well demonstrated its excellent safety and efficacy profile. Very recently, a novel chimeric JEV live vaccine based on the genetic background of Yellow fever virus (YFV) 17D strain was licensed in Australia and is under active consideration for license in Thailand [10].

JEV belongs to the Flavivirus genus in the family Flaviviridae together with YFV, dengue virus (DENV), West Nile virus (WNV), Murray Valley encephalitis virus (MVEV) and tick-borne encephalitis virus (TBEV). The genome of JEV is a positive-sense single-stranded 


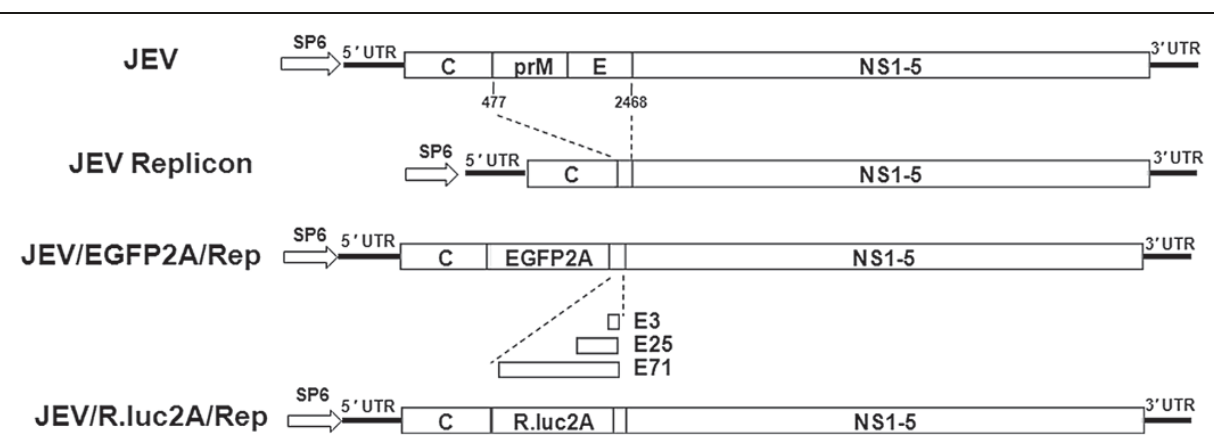

Figure 1 The schematic representation of JEV replicons constructed in this study. The prM/E coding region was deleted from the genome of JEV (SA14-14-2) except for the C-terminal amino acid residues of E protein. FMDV-2A was fused downstream of the EGFP and R.luc reporter genes to ensure cytosolic cleavage of heterologous genes.

RNA molecule comprising 10, 976 nucleotides with a long open reading frame coding for three structural $(\mathrm{C}$, prM, and E) and seven nonstructural (NS1, NS2A, NS2B, NS3, NS4A, NS4B, NS5) proteins. The RNA genome has a type I cap structure at its $5^{\prime}$-end and lacks the poly (A) tail at its $3^{\prime}$-end [11].

A viral replicon is a self-replicating sub-genomic viral RNA originated from viral genome, which contains viral non-structural genes that are critical for viral genome replication with structural proteins deleted or replaced by foreign genes. This non-infectious replicon provides a valuable platform to study the function and structure of viral genome RNA, express foreign proteins and develop novel vaccines. In recent years, many flavivirus replicons have been developed, including Kunjin virus [12], Tick-borne encephalitis virus [13], DENV [14-17], Yellow fever virus [18,19], and West Nile virus [20-23].

\section{A \\ Anti-NS1}


B

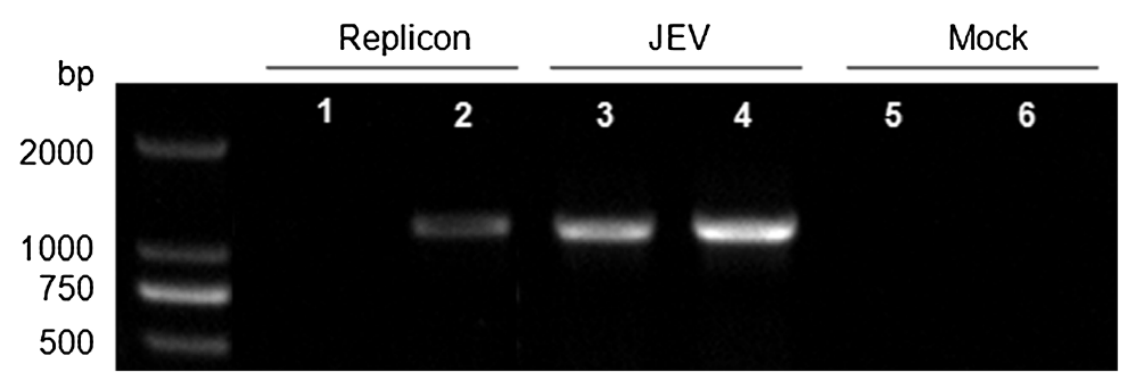

Figure 2 Characterization of the JEV replicon. (A) BHK-21 cells transfected with the JEV replicon pJE3Rep were subjected to indirect immunofluorescence assay (IFA). JEV viral proteins were visualized using NS1- and E-specific mouse monoclonal antibody and stained with fluorescein isothiocyanate (FITC)-conjugated goat anti-mouse lgG at $72 \mathrm{~h}$ post-transfection. The green signals represent FITC-positive cells. Viral RNAs (JEV) and PBS (Mock) were set as controls. (B) RT-PCR targeted at the prM/E genes (Lane 1, 3 and 5) and NS3 genes (Lane 2, 4 and 6). The amplified products for NS3 and prM/E gene were 1185 and 1208 bp, respectively. Total RNAs were extracted from BHK-21 cells at $72 \mathrm{~h}$ post transfection. Viral RNAs (JEV) and PBS (Mock) were set as controls. 
The reverse genetic system of JEV is greatly hampered due to the toxicity of JEV cDNA in bacteria. Despite extensive efforts for many years [24-27], a genetically stable full-length infectious cDNA clone of JEV was not obtained until the bacterial artificial chromosome (BAC) was used as a vector in 2003. Then, several JEV replicons using the BAC vector were constructed based on a Korean JEV strain K87P39 to express the foreign proteins [11]. In this work, we described the preparation of a sub-genomic replicon derived from JEV attenuated strain SA14-14-2, as well as, a series of replicons with Enhanced green fluorescent protein (EGFP) and Renilla luciferase (R.luc) reporter genes were constructed and characterized, respectively. These replicons should be useful for studying many aspects of JEV replication, expressing foreign proteins and developing new vaccines.

The low-copy pACNR vector and E. coli MC1061 were employed in our experiments $[19,20,28]$. All the primers used in this study are listed in Additional file 1: Table S1. Firstly, new restriction enzyme sites (XhoI, BspEI, and $A s c \mathrm{I})$ were introduced into the multiple cloning site of pACNR by fusion PCR with Not I( + )and linker-1(-), resulting in a new vector named pANCR-L1. The $5^{\prime}$-half (nucleotides [nt] 1 to 3446), 3'-1 (3099 to 7299) and 3'-2 (7299 to 10976) fragments covering the full-length JEV SA14-14-2 genomic cDNA were amplified by RT-PCR using high-fidelity M-MLV reverse transcription polymerase (TaKaRa), respectively. Primer pair of F-AscI (+) and J-BspEI (-) were used to amplify the $5^{\prime}$-half fragments encompassing the SP6 promoter. The cDNA product was digested with $A s c \mathrm{I}$ and $B s p \mathrm{EI}$, and then inserted into the $A s c \mathrm{I}$ and BspEI sites of pACNR-L1. Following transformation in competent E. coli MC1061 (Invitrogen), positive clones were selected and confirmed by DNA sequencing and named as pANCR-JEV-5. The $3^{\prime}-1$ and $3^{\prime}-2$ fragments were obtained using the primer



Figure 3 Characterization of the JEV EGFP reporter replicons. BHK-21 cells were transfected with RNA transcripts from linearized pJ/GFP/ E3Rep, pJ/GFP/E25Rep and pJ/GFP/E71Rep, respectively and the expression of EGFP was observed under light and fluorescence microscopy at the indicated time points post transfection. 
pairs of pBRJEV-7289(+), pBRJEV-7289(-) and pBRJEV7289-3U (+), pBRJEV-7289-3U (-), respectively. Both fragments were first subcloned into pGEM-T-Easy vector (TaKaRa), yielding pT-3099-10976. The pT-3099 -10976 was then digested with BspEI and XhoI, and then inserted into the pACNR-L1 to obtain the subclone coding the 3 '-half fragment and named pANCR-JEV-3. Next, the JR-5-F (1 to 477) and JR-5-S (2468 to 2649) fragments were amplified by RT-PCR using primer sets F-AscI, R-J-Rep and F-J-Rep, R-J-BglII (introduced another KasI site by a silent A to $C$ substitution on position 474 of $\mathrm{C}$ gene), and integrated together by overlapping PCR to generate plasmid J-R-5 ( $\Delta$ prM/E). The J-R $-5(\Delta \mathrm{prM} / \mathrm{E})$ fragment was finally digested with Not I and XhoI, and then ligated into the plasmid pANCR-JEV-3, resulting into the desired JEV replicon pJE3Rep. The constructed JEV replicon pJE3Rep lacks the structural protein coding regions except for the whole $C$ protein and the last 3 amino acids of the $E$ protein (Figure 1). It has been demonstrated that the coding sequence of $\mathrm{C}$ protein was important in the RNA cyclization and essential for the replication [29-31]. On the other hand, the last three amino acids of the E protein were retained to ensure proper translocation of the NS1 protein into the lumen of the endoplasmic reticulum [12].

To assess whether the replicon was functional, the plasmid pJE3Rep was linearized by digestion with the XhoI restriction enzyme and then transcribed in vitro by using the RiboMax ${ }^{\mathrm{TM}}$ Large Scale RNA Production system-sp6 Transcription Kit (Promega) in the presence of the $\mathrm{m}^{7} \mathrm{G}\left(5^{\prime}\right)$ ppp $\left(5^{\prime}\right) \mathrm{G}$ cap analogs (Promega). The in vitro synthesized RNAs were transfected into BHK-21 cells with Lipofectamine 2000 (Invitrogen) as previously described [32]. Viral RNAs extracted from JEV infected BHK-21 cells were transfected in the same way and used as a positive control. PBS-transfected cells were used as a negative control (Mock). To make sure whether viral protein were successfully expressed by the replicon, the transfected cells were fixed and analyzed by indirect immunofluorescence assay at $72 \mathrm{~h}$ post transfection using monoclonal antibodies against $\mathrm{E}$ and NS1 proteins of JEV, respectively. As shown in Figure 2A, the negative control did not react with any of the antibodies, and both NS1 and E proteins were detected in the viral RNAs transfected cells. The replicon-transfected cells only reacted with anti-NS1 monoclonal antibody, and no E protein expression was noticed, indicating the JEV replicon could express viral nonstructural protein. Additionally, RT-PCR was carried out for the BHK-21 cells using primers pair F2/R2 and F6/R6 targeting at the $\mathrm{prM} / \mathrm{E}$ and the NS3 genes. As expected, both genes were detected in viral RNAs transfected cells (lane 3, 4), while only the NS3 gene (lane 2) was detected in the JEV replicon transfected cells. No PCR products were amplified for the mock transfected cells as expected (lane 5, 6). These results demonstrated that the constructed JEV replicon could efficiently replicate and express nonstructural protein in transfected cells.

To further adapt the potential application of JEV replicon system, a series of JEV replicons expressing the enhanced green fluorescent protein (EGFP) reporter gene (Figure 1) were constructed. A DNA fragment encoding $2 \mathrm{~A}$ protease of foot-and-mouth disease virus (FMDV-2A) in fusion with the downstream region of the EGFP encoding sequence was amplified from pEGFP-N1 vector (Promega) using primer set F-KAS-EGFP and J-2A-E3-R. The resulting fusion PCR fragment was digested by KasI and $B s p \mathrm{EI}$ and ligated into the pJE3Rep, yielding the JEV reporter-replicon $\mathrm{pJ} / \mathrm{EGFP} 2 \mathrm{~A} / \mathrm{E} 3 \mathrm{Rep}$. Another two JEV reporter-replicons retaining the C-terminal 25 and 71 amino acids residues of $\mathrm{E}$ protein, $\mathrm{pJ} / \mathrm{EGFP} 2 \mathrm{~A} / \mathrm{E} 25 \mathrm{Rep}$ and $\mathrm{pJ} / \mathrm{EGFP} 2 \mathrm{~A} / \mathrm{E} 71$ Rep, respectively, were constructed. All the molecular constructs were prepared by using standard molecular biology techniques, and confirmed by restriction digest analysis and DNA sequencing.

To measure the expression of EGFP reporter gene, equal amounts of RNA transcripts derived from $\mathrm{pJ} /$ EGFP2A/E3Rep, pJ/EGFP2A/E25Rep and pJ/EGFP2A/ E71Rep were transfected into BHK-21 cells. Transient expression of EGFP in transfected cells was visualized under light and fluorescence microscopy at 48, 72, 96 and 120 hours post transfection. As shown in Figure 3,

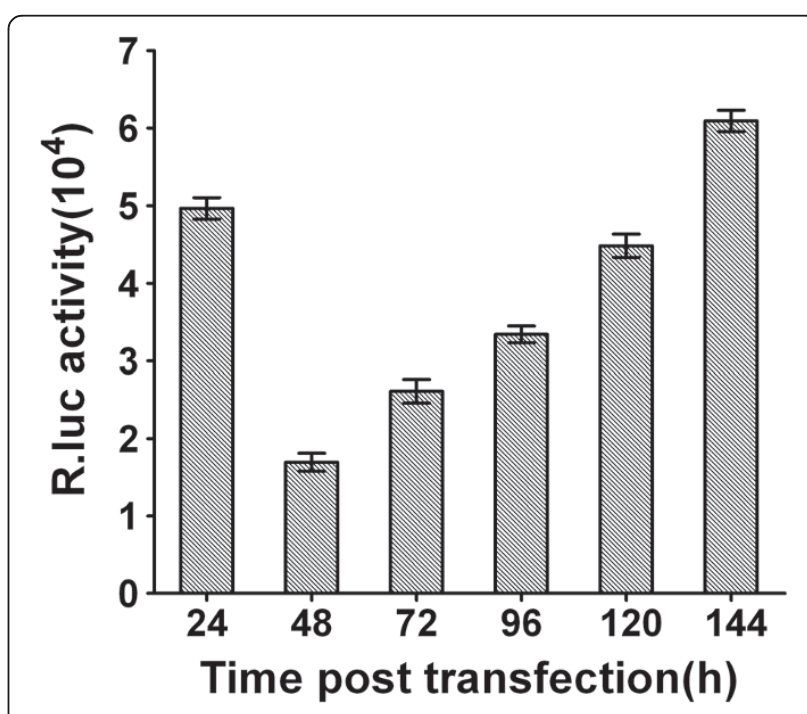

Figure 4 Characterization of the JEV R.Luc reporter replicon. In vitro RNA transcripts of $\mathrm{pJ} /$ R.luc/E3Rep were transfected into BHK21 cells, and the cells were harvested at the indicated times post transfection, the luciferase activity was measured by using a Glomax system (Promega) according to the manufacturer's instruction. The results represent the means and standard deviations derived from three independent experiments performed in triplicate. 
EGFP-positive cells were observed in all the replicontransfected BHK-21 cells, indicating that the reporter gene was successfully expressed in the cells. An increase of EGFP-positive cells could be observed in the early time post transfection, indicating the success of selfreplication of the JEV replicon. Then, the EGFP-positive cells began to reduce at about $120 \mathrm{~h}$ post transfection. The EGFP-positive rate in the pJ/EGFP2A/E3Reptransfected cells was relatively higher than that in $\mathrm{pJ} /$ EGFP2A/E25Rep- and pJ/EGFP2A/E71Rep-transfected cells. And the pJ/EGFP2A/E71Rep replicon showed the lowest positive rate among the three JEV replicons. These results indicated that the remaining amino acid length at the C-terminal of $\mathrm{E}$ protein could potentially affect JEV replication. Additionally, no obvious cytotoxicity was observed for all three replicons under light microscope, which is in agreement with previous results from YFV replicons [19]. Thus, all these JEV EGFP reporter-replicons could replicate efficiently and express EGFP proteins in BHK-21 cells.

Finally, the Renilla luciferase (R.luc) reporter JEV replicon, pJ/R.luc2A/E3Rep, was constructed using the same strategy as the EGFP replicon (Figure 1) and the R.luc gene was amplified from pRL-CMV vector (Promega). To observe the expression of R.luc, in vitro RNA transcripts of $\mathrm{pJ} /$ R.luc/E3Rep were transfected into BHK-21 cells as previously described. For luciferase assay, the transfected cells were lysed in $100 \mu \mathrm{l}$ of passive lysis buffer (Promega) per well of a 48-well plate at $24,48,72,96,120$ and $144 \mathrm{~h}$ post transfection, respectively. Triplicate wells were seeded for each time point. In general, luciferase activity assays were initiated by mixing $20 \mu \mathrm{l}$ of prepared cell extract with $100 \mu \mathrm{l}$ of the appropriate R.luc substrate, and then measured by using a Glomax system (Promega) according to the manufacturer's instruction. The results showed that the R.luc activity firstly peaked at $24 \mathrm{~h}$ post transfection, then declined rapidly from the first peak. After that, the R.luc activity began to rise stably until it reached a second peak at $144 \mathrm{~h}$ post transfection (Figure 4). Luciferase activity at early time points is indicative of translation of the input replicon RNAs, while the second peak at later time points represents proteins expressed by newly synthesized RNAs [33]. Additionally, IFA and RTPCR assays also confirmed that the pJ/R.luc2A/E3Rep efficiently expressed the NS1 proteins and synthesized viral RNA of JEV at $72 \mathrm{~h}$ post transfection (data not shown). These results indicated that this R.luc reporter JEV replicon could replicate efficiently and express R.luc proteins in BHK-21 cells.

Previously, we have generated the infectious clone of JEV and constructed mutant JEV by using reverse genetic technology [34]. In this study, we have constructed a series of JEV replicons based on the JEV live vaccine strain SA14-14-2, and all these replicons are functionally active to replicate and express the desired foreign reporter genes. These replicons constructed herein are under the control of SP6 promoter. Previously, some DNA-based JEV replicons that under the control of CMV promoter have been developed and adapted for pseudo infectious particles $[35,36]$. These JEV replicons not only help to understand the molecular mechanism of viral replication, but also provide a powerful tool for foreign proteins expression, chimeric vaccine and singleround virus like particles (VLP) based vaccine development. The live vaccine virus SA14-14-2 has been widely used in the most JEV endemic countries owing to its highly efficiency, and very few adverse effects [37-39]. Another flavivirus live vaccine strain, YFV 17D, has been widely used as genetic backbone for chimeric flavivirus vaccine development [40-45]. The potential applications of JEV SA14-14-2 in vaccine development are of high significance and deserve further investigation $[11,46,47]$. Currently, we are working with these JEV replicons to generate a series of chimeric flaviviruses vaccine candidates.

\section{Additional file}

Additional file 1: Table S1. Oligonucleotide primers for construction of JEV replicons and inserting reporter genes.

\section{Competing interests}

The authors declare that they have no competing interests.

\section{Authors' contributions}

SHL and XFL carried out most of the experiments and drafted the manuscript. HZ, YQD, XDY, SHZ, JT, QY, EDQ participated in experiments and data analysis. CFQ designed the study, supervised the work and edited the final version of this manuscript. All authors have read and approved the final version of the manuscript.

\section{Acknowledgements}

We thank Chengdu Institute of Biological Products for providing the work seed of JEV live vaccine SA14-14-2. This work was supported in part by the National Science and Technology Major Project of China (2013ZX10004-805), National Basic Research Project of China (2012CB518904) and National Natural Science Foundation of China (81101243 and 31270974).

Received: 10 October 2012 Accepted: 22 February 2013

Published: 26 February 2013

\section{References}

1. Kumar R, Tripathi P, Singh S, Bannerji G: Clinical features in children hospitalized during the 2005 epidemic of Japanese encephalitis in uttar pradesh, India. Clin Infect Dis 2006, 43:123-131.

2. Murgod UA, Muthane UB, Ravi V, Radhesh S, Desai A: Persistent movement disorders following Japanese encephalitis. Neurology 2001, 57:2313-2315.

3. Misra UK, Kalita J: Overview: Japanese encephalitis. Prog Neurobio/ 2010, 91:108-120.

4. Erlanger TE, Weiss S, Keiser J, Utzinger J, Wiedenmayer K: Past, present, and future of Japanese encephalitis. Emerg Infect Dis 2009, 15:1-7.

5. Endy TP, Nisalak A: Japanese encephalitis virus: ecology and epidemiology. Curr Top Microbiol Immunol 2002, 267:11-48.

6. Mackenzie JS, Johansen CA, Ritchie SA, van den Hurk AF, Hall RA: Japanese encephalitis as an emerging virus: the emergence and spread of 
Japanese encephalitis virus in Australasia. Curr Top Microbiol Immunol 2002, 267:49-73.

7. Yun SI, Choi YJ, Song BH, Lee YM: 3/ cis-acting elements that contribute to the competence and efficiency of Japanese encephalitis virus genome replication: functional importance of sequence duplications, deletions, and substitutions. J Virol 2009, 83:7909-7930.

8. Oya A, Kurane I: Japanese encephalitis for a reference to international travelers. J Travel Med 2007, 14:259-268.

9. Halstead SB, Thomas SJ: New Japanese encephalitis vaccines: alternatives to production in mouse brain. Expert Rev Vaccines 2011, 10:355-364.

10. Fischer M, Lindsey N, Staples JE, Hills S: Japanese encephalitis vaccines: recommendations of the advisory committee on immunization practices (ACIP). MMWR Recomm Rep 2010, 59:1-27.

11. Yun SI, Choi YJ, Yu XF, Song JY, Shin YH, Ju YR, Kim SY, Lee YM: Engineering the Japanese encephalitis virus RNA genome for the expression of foreign genes of various sizes: implications for packaging capacity and RNA replication efficiency. J Neurovirol 2007, 13:522-535.

12. Khromykh AA, Westaway EG: Subgenomic replicons of the flavivirus Kunjin: construction and applications. J Virol 1997, 71:1497-1505.

13. Gehrke R, Ecker M, Aberle SW, Allison SL, Heinz FX, Mandl CW: Incorporation of tick-borne encephalitis virus replicons into virus-like particles by a packaging cell line. J Virol 2003, 77:8924-8933.

14. Mosimann AL, de Borba L, Bordignon J, Mason PW, dos Santos CN: Construction and characterization of a stable subgenomic replicon system of a Brazilian dengue virus type 3 strain (BR DEN3 290-02). J Virol Methods 2010, 163:147-152.

15. Suzuki R, de Borba L, Santos CN Dd, Mason PW: Construction of an infectious CDNA clone for a Brazilian prototype strain of dengue virus type 1: characterization of a temperature-sensitive mutation in NS1. Virology 2007, 362:374-383.

16. Ng CY, Gu F, Phong WY, Chen YL, Lim SP, Davidson A, Vasudevan SG: Construction and characterization of a stable subgenomic dengue virus type 2 replicon system for antiviral compound and siRNA testing. Antiviral Res 2007, 76:222-231.

17. Pang $X$, Zhang M, Dayton Al: Development of Dengue virus type 2 replicons capable of prolonged expression in host cells. BMC Microbiol 2001, 1:18

18. Corver J, Lenches E, Smith K, Robison RA, Sando T, Strauss EG, Strauss JH: Fine mapping of a cis-acting sequence element in yellow fever virus RNA that is required for RNA replication and cyclization. J Virol 2003, 77:2265-2270.

19. Jones CT, Patkar CG, Kuhn RJ: Construction and applications of yellow fever virus replicons. Virology 2005, 331:247-259.

20. Rossi SL, Zhao Q, O'Donnell VK, Mason PW: Adaptation of West Nile virus replicons to cells in culture and use of replicon-bearing cells to probe antiviral action. Virology 2005, 331:457-470.

21. Shi PY, Tilgner M, Lo MK: Construction and characterization of subgenomic replicons of New York strain of west nile virus. Virology 2002, 296:219-233.

22. Yamshchikov VF, Wengler G, Perelygin AA, Brinton MA, Compans RW: An infectious clone of the west nile flavivirus. Virology 2001, 281:294-304.

23. Moritoh K, Maeda A, Nishino T, Sasaki N, Agui T: Development and application of west nile virus subgenomic replicon RNA expressing secreted alkaline phosphatase. J Vet Med Sci 2011, 73:683-686.

24. Mishin VP, Cominelli F, Yamshchikov VF: A 'minimal' approach in design of flavivirus infectious DNA. Virus Res 2001, 81:113-123.

25. Sumiyoshi $\mathrm{H}$, Hoke CH, Trent DW: Infectious Japanese encephalitis virus RNA can be synthesized from in vitro-ligated cDNA templates. J Virol 1992, 66:5425-5431.

26. Sumiyoshi $\mathrm{H}$, Tignor $\mathrm{GH}$, Shope RE: Characterization of a highly attenuated Japanese encephalitis virus generated from molecularly cloned cDNA. J Infect Dis 1995, 171:1144-1151.

27. Zhang F, Huang Q, Ma W, Jiang S, Fan Y, Zhang H: Amplification and cloning of the full-length genome of Japanese encephalitis virus by a novel long RT-PCR protocol in a cosmid vector. J Virol Methods 2001, 96:171-182

28. Yoshii K, Holbrook MR: Sub-genomic replicon and virus-like particles of Omsk hemorrhagic fever virus. Arch Virol 2009, 154:573-580.

29. Alvarez DE, Lodeiro MF, Luduena SJ, Pietrasanta LI, Gamarnik AV: Long range RNA-RNA interactions circularize the dengue virus genome. J Virol 2005, 79:6631-6643.
30. Bredenbeek PJ, Kooi EA, Lindenbach B, Huijkman N, Rice CM, Spaan WJ: A stable full-length yellow fever virus cDNA clone and the role of conserved RNA elements in flavivirus replication. J Gen Virol 2003, 84:1261-1268.

31. Khromykh AA, Meka H, Guyatt KJ, Westaway EG: Essential role of cyclization sequences in flavivirus RNA replication. J Virol 2001, 75:6719-6728.

32. Cao F, Li XF, Yu XD, Deng YQ, Jiang T, Zhu QY, Qin ED, Qin CF: A DNAbased west nile virus replicon elicits humoral and cellular immune responses in mice. J Virol Methods 2011, 178:87-93.

33. Patkar CG, Larsen M, Owston M, Smith JL, Kuhn RJ: Identification of inhibitors of yellow fever virus replication using a replicon-based highthroughput assay. Antimicrob Agents Chemother 2009, 53:4103-4114.

34. Ye Q, Li XF, Zhao H, Li SH, Deng YQ, Cao RY, Song KY, Wang HJ, Hua RH, Yu $Y X$, et al: A single nucleotide mutation in NS2A of Japanese encephalitislive vaccine virus (SA14-14-2) ablates NS1' formation and contributes to attenuation. J Gen Virol 2012, 93:1959-1964.

35. Huang Y, Liu S, Yang P, Wang C, Du Y, Yu W, Sun Z: Replicon-based Japanese encephalitis virus vaccines elicit immune response in mice. J Virol Methods 2012, 179:217-225.

36. Huang Y, Liu S, Yang P, Wang C, Du Y, Sun Z, Yu W: Influence of Japanese enciphalitis virus capsid protein on the self-replicate ability of JEV replicon vectors. Sheng Wu Gong Cheng Xue Bao 2010, 26:1088-1094.

37. Hennessy S, Liu Z, Tsai TF, Strom BL, Wan CM, Liu HL, Wu TX, Yu HJ, Liu QM, Karabatsos N, et al: Effectiveness of live-attenuated Japanese encephalitis vaccine (SA14-14-2): a case-control study. Lancet 1996, 347:1583-1586.

38. Tandan JB, Ohrr H, Sohn YM, Yoksan S, Ji M, Nam CM, Halstead SB: Single dose of SA 14-14-2 vaccine provides long-term protection against Japanese encephalitis: a case-control study in Nepalese children 5 years after immunization. Vaccine 2007, 25:5041-5045.

39. Ohrr H, Tandan JB, Sohn YM, Shin SH, Pradhan DP, Halstead SB: Effect of single dose of SA 14-14-2 vaccine 1 year after immunisation in Nepalese children with Japanese encephalitis: a case-control study. Lancet 2005, 366:1375-1378.

40. Franco D, Li W, Qing F, Stoyanov CT, Moran T, Rice CM, Ho DD: Evaluation of yellow fever virus 17D strain as a new vector for HIV-1 vaccine development. Vaccine 2010, 28:5676-5685.

41. McGee CE, Lewis MG, Claire MS, Wagner W, Lang J, Guy B, Tsetsarkin K, Higgs S, Decelle T: Recombinant chimeric virus with wild-type dengue 4 virus premembrane and envelope and virulent yellow fever virus Asibi backbone sequences is dramatically attenuated in nonhuman primates. J Infect Dis 2008, 197:693-697.

42. Chambers TJ, Liang Y, Droll DA, Schlesinger JJ, Davidson AD, Wright PJ, Jiang $X$ : Yellow fever virus/dengue- 2 virus and yellow fever virus/ dengue- 4 virus chimeras: biological characterization, immunogenicity, and protection against dengue encephalitis in the mouse model. $J$ Virol 2003, 77:3655-3668.

43. van Der Most RG, Murali-Krishna K, Ahmed R, Strauss JH: Chimeric yellow fever/dengue virus as a candidate dengue vaccine: quantitation of the dengue virus-specific CD8 T-cell response. J Virol 2000, 74:8094-8101.

44. Guirakhoo F, Weltzin R, Chambers TJ, Zhang ZX, Soike K, Ratterree M, Arroyo J, Georgakopoulos K, Catalan J, Monath TP: Recombinant chimeric yellow fever-dengue type 2 virus is immunogenic and protective in nonhuman primates. J Virol 2000, 74:5477-5485.

45. Guirakhoo F, Zhang ZX, Chambers TJ, Delagrave S, Arroyo J, Barrett AD, Monath TP: Immunogenicity, genetic stability, and protective efficacy of a recombinant, chimeric yellow fever-Japanese encephalitis virus (ChimeriVax-JE) as a live, attenuated vaccine candidate against Japanese encephalitis. Virology 1999, 257:363-372.

46. Chambers TJ, Droll DA, Jiang X, Wold WS, Nickells JA: JE Nakayama/JE SA14-14-2 virus structural region intertypic viruses: biological properties in the mouse model of neuroinvasive disease. Virology 2007, 366:51-61.

47. Yun SI, Song BH, Koo Y, Jeon I, Byun SJ, Park JH, Joo YS, Kim SY, Lee YM: Japanese encephalitis virus-based replicon RNAs/particles as an expression system for HIV-1 Pr55 Gag that is capable of producing viruslike particles. Virus Res 2009, 144:298-305.

doi:10.1186/1743-422X-10-64

Cite this article as: Li et al:: Development and characterization of the replicon system of Japanese encephalitis live vaccine virus SA14-14-2. Virology Journal 2013 10:64. 